\title{
Activity in the dorsomedial striatum decreases with improvement in motor coordination
}

Stefano Cataldi ${ }^{1}$, Clay Lacefield ${ }^{1,2}$, Shashaank N ${ }^{3}$, Gautam Kumar ${ }^{4}$, David Sulzer ${ }^{1,5,6}$

${ }^{1}$ Departments of Psychiatry, Columbia University: Division of Molecular Therapeutics, New York

State Psychiatric Institute, New York, NY 10032

${ }^{2}$ Division of Systems Neuroscience, New York State Psychiatric Institute, New York, NY 10032

${ }^{3}$ Department of Computer Science, Columbia University, Schapiro Center for Engineering and Physical Science Research, New York, NY 10027

${ }^{4}$ Neuroscience Program, University of Maryland School of Medicine, Baltimore, MD 21201

${ }^{5}$ Departments of Neurology, Columbia University, New York, NY 10032

${ }^{6}$ Department of Pharmacology, Columbia University, New York, NY 10032

*Correspondence author: $\underline{\text { ds43@ cumc.columbia.edu }}$

Key words: striatum, basal ganglia, motor learning, direct pathway, treadmill, deeplabcut

\begin{abstract}
It is widely thought that during early stages of motor learning, the dorsomedial striatum facilitates the learning of goal-directed actions, and at later stages, the learned actions are transferred to the dorsolateral striatum, which enables motor actions to become a skill or habit. It is however unknown if these striatal regions are simultaneously active as expertise is acquired during practice. To address this question, we developed a treadmill task to track changes in mouse locomotor coordination during practice running at a range of speeds. We analyzed body position and paw movement to evaluate changes in motor coordination over practice using DeepLabCut and custombuilt code. By simultaneous evaluation of motor coordination improvements and fiber photometry recordings of neuronal calcium activity during training, we found that direct pathway dorsomedial striatum neurons exhibited reduced activity as the mouse became proficient at running on the treadmill. In contrast, direct pathway activity in dorsolateral striatum was similar throughout training and did not correlate with increased skill proficiency. These results provide new tools to measure changes in fine motor skills during simultaneous recordings of brain activity, revealing fundamental features of the neural substrates of motor learning.
\end{abstract}




\section{Introduction}

Synaptic pathways in the striatum are central to basal ganglia functions, including motor control and learning and organization of motor skills. The development of techniques to record neuronal activity of specific populations and animal models of motor disorders using pharmacological or genetic manipulations has revealed new details about the pathways that underlie motor performance and motor learning. The dorsal portion of the striatum has been found to be particularly important for expressing automatic fine skills and micromovements embedded in actions (Yin et al., 2009). In rodents, this region is typically divided into two main portions: the dorsomedial striatum (DMS) and the dorsolateral striatum (DLS), corresponding to the human caudate and putamen respectively. The DMS receives afferents from prefrontal and associative cortices, while the DLS mostly receives input from sensorimotor cortical areas (Cataldi, Stanley, Miniaci, \& Sulzer, 2021; Graybiel, 2008; Hunnicutt et al., 2016).

It was previously shown by in vivo electrophysiological recordings that the DMS is engaged in early training on a rotarod (Lingawi \& Balleine, 2012; Yin et al., 2009), a task commonly used to study motor performance, while the DLS is particularly active during late training. However, single unit recording during extended training on a lever press task showed a similar activity pattern between DLS and DMS once the skill was acquired (Vandaele et al., 2019).

Spiny projection neurons (SPNs) constitute the majority of striatal neurons (Silberberg \& Bolam, 2015) and are generally classified as D1 dopamine receptor expressing SPNs that form the direct pathway (D1-SPNs) and D2 dopamine receptor expressing SPNs that form the indirect pathway (D2-SPNs; Gangarossa et al., 2013; Gerfen et al., 1990; Kawaguchi, Wilson, \& Emson, 1990; Wall, DeLaParra, Callaway, \& Kreitzer, 2013). D1-SPNs axons project to the basal ganglia output structures, the globus pallidus internal segment and substantia nigra pars reticulata, while D2-SPNs project to the globus pallidus external segment, thus making indirect connection with output nuclei (Albin, Young, \& Penney, 1989; DeLong, 1990). According to these classical models, activation of the direct pathway releases neurons in the motor thalamus from inhibition, thus promoting movement. Consistent with these models, optogenetic activation of the D1-SPNs increases locomotion and reduces freezing (Kravitz et al., 2010).

The activity of D1-SPNs has been studied at the level of the initiation, termination, and velocity of a specific motor action (Jin, Tecuapetla, \& Costa, 2014), but few studies have investigated the role of these neurons during steps involved in motor learning (Cataldi et al., 2021). 
Computational analysis of behavioral and physiological data can provide new means to interpret complex motor actions, such as running, across multiple conditions and over time.

Here we introduce a paradigm to detect fine changes in motor coordination as a mouse acquires the ability to run on a motorized treadmill, while simultaneously recording activity of direct pathway neurons in the DLS or DMS, in order to study the neural and behavioral changes that occur across early and late stages of learning. We combine a well-established pose tracking method, DeepLabCut (Mathis et al., 2018), with custom-built Python-based software to analyze and interpret the behavioral data. We then correlate the results with changes in calcium activity measured by fiber photometry within the different striatal subregions over training. Our results indicate that DMS activity is reduced over training, while DLS activity is similar during both early and late stages of learning.

\section{Materials and Methods}

\section{Animals}

Wild-type and transgenic mice expressing Cre-recombinase in D1-expressing SPNs (D1-cre) were used for these experiments. All animal procedures were approved by the animal care and use committee of the New York State Psychiatric Institute. Experiments were performed on 3 to 6month-old male and female mice. C57BL/6 (000664) from Jackson Laboratory were used as control animals. D1-Cre transgenic mice were obtained from MMRC/Jax (ey262 D1-cre tg-drdlacre) and crossed with C57BL/6 mice.

\section{Viral expression of GcAMP6f and fiber implants}

To achieve Cre-dependent expression of GCaMP6f in direct pathway SPNs, AAV vectors containing Flex-GCaMP6f (AAV.9.Syn.Flex.GCaMP6f.WPRE.SV40, Addgene; TIter $\geq 2.1 \times 13 \mathrm{GC} / \mathrm{mL}$ ) were injected into the right DLS or DMS by stereotaxic surgery. During the surgery, a small skull craniotomy $(1 \mathrm{~mm} \times 1 \mathrm{~mm})$ above the injection site was opened with a dental drill. A glass pipette attached to a Nanoject II (Drummond Scientific) was filled with the AAV GCaMP6f and lowered to target locations in the dorsomedial and dorsolateral striatum (tip coordinates from bregma: AP -0.5mm, ML +2.5 DV -3.2mm for DLS, AP +1.2mm ML -0.8mm DV -2.8mm DMS). A total volume of $150 \mathrm{~nL}$ AAV vector per site was injected over 10 minutes. The pipette was left in place for 5 more minutes before removal. An optic fiber (Doric Lenses; 
MFC 300/370-0.22_4mm_MF2.5_FLT) was subsequently lowered to the same coordinates. The skull was then covered with dental acrylic to secure the optic fiber in place. Alternatively, optofluid cannulas (Doric Lenses; OmFC_MF1.25_300/370-0.22_4.2_FLT_4) were implanted for simultaneous GCaMP6f recording and local microinjection of drug or saline. Animals were allowed to recover, and, for optimal viral expression, photometry experiments were performed 4 weeks after surgery. Three to five days before running on the treadmill, animals were placed in an open field chamber to record a baseline calcium signal and habituate the animals to the tethered optical fiber.

\section{Custom-built treadmill}

In order to precisely control the animal's locomotion, mice were placed on a custom motorized treadmill (Supplementary Figure 1A). The treadmill consisted of a 1m clear belt stretched between two 3" diameter acrylic wheels on an aluminum frame (8020.net). Treadmill speed was controlled by an Arduino-based system (OpenMaze.org, OM4 board) that adjusted the speed of a $12 \mathrm{~V}$ gear motor attached to the axle of one of the treadmill wheels through pulse-width modulation (PWM). Belt speed was measured using a quadrature rotary encoder (Digikey \#ENS1J) attached to the other axle and decoded by the Arduino. The Arduino/OpenMaze setup was also used to send synchronization pulses to coordinate behavior with video recording and fiber photometry. Mouse movement on the treadmill was constrained by placing the mouse into a 6" long clear acrylic box over the center of the treadmill and covering the entire width of the belt, which forced the animal to walk on the moving belt to avoid being forced into the back wall during belt movement. An acrylic mirror was fixed at a $45^{\circ}$ angle under the clear acrylic box and mesh belt to allow high speed videography of the animal's locomotion from lateral and ventral viewpoints simultaneously.

\section{Treadmill testing}

Mice undergoing experimental procedures were weighed prior to testing. All tests were performed in the morning between 9:30 am and 1:30 pm. All animals were assessed at 4-6 months of age, and all experimentation and analysis were conducted with the experimenter blinded to genotype. After 3-day familiarization to experimenter handling, mice underwent the following training paradigm. 
Mice were placed on the treadmill within the clear inbuilt open box and were left free to explore the environment for 30 seconds. After this 30 second baseline, the treadmill was activated through the Arduino system at a low speed of 3m/min., with an increment every 60 seconds up to $12 \mathrm{~m} / \mathrm{min}$ (total of 5 speeds; intermediate speeds are $6 \mathrm{~m} / \mathrm{min}, 8 \mathrm{~m} / \mathrm{min}$, and $10 \mathrm{~m} / \mathrm{min}$; Figure 1A). After 5 minutes of running, the treadmill was turned off for a final baseline recording of 30 seconds. Finally, animals were removed from the treadmill and placed back into their home cage. This process is then repeated for 12 consecutive days, including home cage baseline recording and 30 seconds on the treadmill with the motor off, before and after the running time. Animal weight was recorded every day before training and showed no significant changes (Supplementary Figure 1B). The Arduino source code is freely available at github.com/DSulzerLab/treadmill.

\section{Photometry recording}

Calcium signals were recorded using a commercial fiber photometry apparatus (Doric Lenses). The system consisted of a console connected to a computer, a four-channel programmable LED driver, two LEDs at 405 and $465 \mathrm{~nm}$, connected to fluorescence dichroic mini cubes and photometric multipliers tubes (PMTs). The $405 \mathrm{~nm}$ wavelength is the isosbestic point for GCaMP,

a point where fluorescence does not change depending on calcium concentration (Supplementary Figure 2A). Detection at this wavelength was used to remove background noise (movement artifact or GCaMP auto-fluorescence). The $465 \mathrm{~nm}$ excitation provides detection of GCaMP signal where the intensity of fluorescence is proportional to cytosolic calcium concentration.

Calcium signals from all animals were recorded for 5 minutes as the mice explored their home cage prior to treadmill testing. This signal is used as a baseline to evaluate whether there is any loss of signal over the several days of testing, as well as to habituate the animal to be connected to the optical fiber.

\section{Calcium signal analysis}

Calcium signals were processed using custom-built Python code to remove background noise and detect individual calcium peaks. The process consisted of 1) down sampling the signal to 30 samples/sec to match the sampling rate of behavioral recording, 2) normalization of the data and removal of background noise, and 3) identification and quantification of peak events (peaks count 
and peak amplitude). The source code is freely available at github.com/DSulzerLab/Calcium_peaks_analysis.

\section{Video Analysis}

Behavioral experiments were recorded using a high speed USB webcam (Sony PS3eye) and Kinovea video acquisition software, synchronized to the Arduino/OpenMaze behavior system with an infrared LED, and analyzed post-hoc using DeepLabCut (Mathis et al., 2018) to track individual paw movement (the 4 paws, head, rear of the body, and tail were labeled; data was processed using Google Colaboratory; approximately 30,000-40,000 iterations were sufficient for good quality tracking).

The DeepLabCut results were later processed with custom-built R code to compute the step length of each paw, and custom-built Python code to return a "coordination score" from 1 to 5, with 1 being poor coordination and 5 being excellent coordination. The coordination score was computed using three metrics: the expected value of the mouse head's $\mathrm{X}$ axis position, the standard deviation (SD) of the mouse head's $\mathrm{Y}$ axis position, and the average step length of the four individual paws. First, the expected value of the mouse head's X axis position from 1 (frontmost) to 5 (backmost) was computed by dividing the box into five quadrants (Figure 1B) and calculating the probability of the head being positioned in a certain quadrant. Second, the standard deviation of the mouse head's Y axis position was computed to identify the sideways movement. Third, the step length was acquired using the R package "train" which uses spatiotemporal animal tracking data to estimate parameters such as the length of each step, straightness (running along a straight line), and speed. In the Python code, the average step length for each paw was computed, and the "paw stability" is determined by calculating the step length's distance from the median of the average step length measurements. Along with the paw stability, the percentiles of these expected value and the $\mathrm{Y}$ axis SD were computed, and these three values were given a weightage of 20/40/40, respectively. The percentiles of the weighted average were scaled between 1 to 5 to arrive at the final coordination score.

Manual scoring of the videos under blinded conditions confirmed the validity and reproducibility of the test and analysis method. The source code is freely available at github.com/DSulzerLab/treadmill. 


\section{Immunohistochemistry}

Once finished with behavioral experiments, mice were terminally anesthetized (euthasol $240 \mathrm{mg} / \mathrm{kg}, 200 \mu \mathrm{L}$ i.p.) and intracardially perfused with PBS then $4 \%$ paraformaldehyde (PFA). Brains were extracted and post fixed overnight $\left(4 \%\right.$ PFA, $\left.4^{\circ} \mathrm{C}\right)$. Coronal slices $(100 \mu \mathrm{m})$ were obtained by vibratome (Leica VT 1200). Sections were rinsed with $0.6 \%$ Triton-X in 1x PBS (PBST; 6x60 min) and blocked in 10\% normal donkey serum (NDS) in PBST (60 min, RT). Primary antibodies: chicken polyclonal GFP (ab13970 Abcam; 1:500) and rabbit polyclonal antityrosine hydroxylase (TH; ab152 Abcam; 1:500), were applied in 2\% NDS in PBST (48 h $\left.4^{\circ} \mathrm{C}\right)$ prior to washing (6x60min PBST) and secondary incubation with species specific Alexafluor IgG secondary antibodies (60 min RT, Invitrogen; 1:500). Tissues were washed again in 0.1\% PBST (6 x 60min), then mounted using DAPI Fluoromount-G® (0100-20, SouthernBiotech). Images were acquired using a 20x oil objective on an Olympus microscope. Sample images in Supplementary Figure 3.

\section{Statistics and data reporting}

Data are presented throughout as mean \pm SEM where $n$ is the number of animals. Comparisons were conducted by 1- or 2-way ANOVA with appropriate post-hoc tests detailed in the text, using Prism 6.0 (GraphPad, San Diego California USA).

\section{Results}

\section{Treadmill running}

To explore motor coordination learning during locomotion, we designed a custom-made motorized treadmill that provides detection of fine improvement in paw mobility (Supplementary Figure 1A and Figure 1B). Our custom-built treadmill is composed of a running belt connected to a motor that runs at a range of velocities. The mouse is placed on the transparent belt and paw movement is tracked through a mirror place below the apparatus that allows visualization of the four paws of the mouse anywhere within the constraining box (detailed in Methods).

A cohort of wild-type (WT) mice were initially examined. On the first day of the protocol, animals were placed on the treadmill and allowed to freely explore the environment for 30 seconds. After this 30 second baseline, the treadmill was activated through the Arduino system at a low speed of $3 \mathrm{~m} / \mathrm{min}$, with an increment every 60 seconds up to $12 \mathrm{~m} / \mathrm{min}$. After 5 minutes of running, 
the treadmill was turned off for a final baseline recording of 30 seconds (Figure 1A). No difference in behavior (or calcium signals) was found between the 30 second periods before and after the running time, and so data were merged and reported as "off time." This protocol is repeated for 12 consecutive days to track changes in the mouse running motor coordination.

The videos were analyzed with the machine-learning based videographic analysis platform DeepLabCut (DLC, Mathis et al., 2018), which allowed us to track paw movements in space and time. DLC provides $\mathrm{X}$ and $\mathrm{Y}$ coordinates of the $2 \mathrm{D}$ plane of the video for each body part of interest across individual frames (Figure 1B \& C). Results obtained by DLC were processed through custom-built R and Python scripts to compute a "motor coordination score" (see Methods) using variables such as the mouse head's position within the box quadrants ( $\mathrm{X}$ axis), the mouse's sideways movement along the treadmill ( $\mathrm{Y}$ axis $\mathrm{SD}$ ), and the mouse paw coordination on the treadmill (average step length). The expected value of the mouse head's X axis position within the box quadrants was used to identify instances in which the mouse was falling behind and was unable to keep up with the moving tape; well-practiced mice tend to run closer to the frontmost quadrant. The Y axis SD was used to identify instances in which the mouse was unable to run along a straight line; well-practiced mice tend to have a lower SD (data not shown). The step length mean for each paw indicates the average distance in pixels between each step. In the initial days of training, naïve mice tend to have a lower motor coordination score (Figure 1E), as they have never been exposed to the treadmill before and have poor coordination during running. These mice would tend to fall behind and tend to make longer steps trying to catch up with the running tape. Moreover, naïve animals run sideways rather than along a straight line. On later days of training, as mice get more competent in running and coordination improves, the score becomes higher (Figure 1E). Control animals trained on the treadmill show a higher coordination score within the fourth day of training. By day 4, all mice performed significantly better than on the first training day (Figure 1E; 1-way ANOVA $* p<0.05)$.

\section{D1-SPNs Dorsomedial and dorsolateral calcium signaling during running}

We then explored the role of the direct pathway in skill acquisition by recording calcium signals from D1-SPNs using D1-Cre mice injected with the GCaMP6f fluorescent calcium sensor in either the DMS or DLS. The animals were first habituated to the optical patch cord through a baseline period recording while exploring their home cage. Treadmill experiments were performed daily 
for 12 consecutive days, as shown for the control animals (Figure 1). Baseline calcium activity was recorded daily for 5 minutes prior to treadmill testing to ensure stability of calcium signals. Recording of baseline D1-SPNs activity in the DMS show no significant changes over the 12 days (Figure 2B; 1-way ANOVA $p=0.28$ ). Similarly, no changes were found in D1-SPNs activity in the DLS (1-way ANOVA $p=0.52$ ).

On each day, following the 5 minutes habituation period, mice were placed on the treadmill and underwent the protocol described above (Figure1A). The injected animals' treadmill performance was similar to control animals (Supplementary Figure 2; 1-way ANOVA * $p<0.05$, for DMS mice and $* * p<0.01$ for DLS mice). We then correlated brain activity with the coordination score to examine neural changes as the mice become proficient at running on the motorized treadmill.

On day 1 of training, all mice show similar average calcium events amplitude between the running time (on-time; independently of the speed of the treadmill) and the time in which the treadmill was off (off-time; 30 seconds prior to running and 30 seconds after; Figure 2C.i. 1-way ANOVA $p=0.43$, Bonferroni's multiple comparisons test $p=0.14$ DLS on-time vs DLS off-time; 1-way ANOVA $p=0.43$, Bonferroni's multiple comparisons test $p=0.79$ DMS on-time vs DMS off-time). Similarly, event counts were comparable between baseline recording and while on the treadmill (on-time and off-time, Supplementary Figure 1C \& D; 2-way ANOVA Time x Column Factor $p=0.82$ for DMS and $p=0.77$ for DLS). Interestingly, DMS D1-SPNs calcium signals showed a significant decrease in overall event amplitude on day 3 while running (on-time) compared to off-time (Figure 2C.ii.; 1-way ANOVA $p<0.01$, Bonferroni's multiple comparisons test $*_{p}<0.05$ DMS on-time vs DMS off-time), which remained consistent throughout the 12 days of testing (Figure 2C.iii. \& iv.; 1-way ANOVA $p<0.01$, Bonferroni's multiple comparisons test ${ }^{*} p<0.05$ DMS on-time vs DMS off-time for day 7; 1-way ANOVA $p<0.0001$, Bonferroni's multiple comparisons test $* * p<0.01$ DMS on-time vs DMS off-time for day 12). Peak counts do not show the same trend (Supplementary Figure 1C; 2-way ANOVA Time x Column Factor $p=0.82$ ).

Conversely, D1-SPN calcium signals recorded from the DLS showed no significant difference in either average amplitude or number of events per minute between running time and off-time over training, (Figure 2C; day 03: 1-way ANOVA $p<0.01$, Bonferroni's multiple comparisons test $p=0.06$ DLS on-time vs DLS off-time; day07: 1-way ANOVA $p<0.01$, 
Bonferroni's multiple comparisons test $p=0.63$ DLS on-time vs DLS off-time; day 12: 1-way ANOVA $p<0.0001$, Bonferroni's multiple comparisons test $p=0.95$ DLS on-time vs DLS off-time; Supplementary Figure 1D, 2-way ANOVA Time x Column Factor $p=0.77$ for DLS).

\section{Discussion}

Current knowledge of motor learning comes from studies analyzing simple tasks, such as lever presses to achieve a food or drug reward or counting the number of falls from a motorized rotarod, which may not be applicable to learning complex skills, such as changes in motor coordination during practice, a feature of activities ranging from athletic tasks to performance on a musical instrument. We suggest that the experimental set-up introduced here provides the means to analyze the improvement of motor abilities during practice and the contribution of specific neural pathways to the task, leading to a more profound understanding of motor learning and execution. Here, we record activity of the direct pathway in the medial and lateral portions of the dorsal striatum while analyzing complex motor activity in freely moving animals in a carefully controlled locomotor task. We developed a computational method to interpret multi-limb behavioral data and identify patterns of motor function that contribute to the improvement of coordination of a running mouse during practice. We found that that DMS and DLS activity progresses differently as the animals gain skill in a motor task.

We found that the rate of calcium signal events in the DMS does not change over training. This is consistent with a report by Mark Wightman et al. that demonstrated distinct active and silent regions in the striatum that that exhibit different activity patterns as a task is acquired (Owesson-White et al., 2016). We note that calcium signals detected by fiber photometry describe bulk activity of a multitude of neurons averaged together, suggesting that synaptic refinement may explain why D1-SPNs activity in the DMS is reduced in amplitude but not in the number of events (Bamford, Wightman, \& Sulzer, 2018). Future experiments may explore this theory by examining single cell activity over training using imaging techniques that utilize miniature head-mounted microscopes.

Given the complexity of the circuits involved in motor learning and the multiple variables of each task, care is needed when interpreting the animal behavior and the interaction between the different regions. Dopamine neurons display different patterns of activation in different behavioral tasks, e.g., in head fixed vs. freely moving animals or depending on whether a reward is present 
(Coddington \& Dudman, 2019) and the activity of striatal neurons may be influenced by similar considerations (Cataldi et al., 2021). We note that the treadmill protocol could encompass aspects of aversion, as the mouse attempts to avoid hitting the back of the box, although we observed no change in calcium signals when the mouse was placed in the box, arguing against a classical fear response. A similar approach could be developed to examine spontaneous running on a wheel, which would avoid box confinement.

More broadly, we suggest that this paradigm provides a means to evaluate motor ability in healthy animals and disease models and will be useful for the evaluation of motor disorders and motor learning deficits due to neurodevelopmental disease, drug dependence, neurotoxic regimens, stroke, seizures, or injury, and to assist in the development of more efficient therapies for these disorders.

\section{Acknowledgements}

We sincerely thank the efforts of Dr. Moshe Shalev and the staff at the Division of Comparative Medicine, Columbia University, for advice and training in animal husbandry.

\section{Competing Interests}

None to declare.

\section{Contributions}

SC conceptualization, project design and administration, investigation, data curation, formal analysis, writing-original draft, editing CL conceptualization, project design and administration, writing-original draft, editing

SN data processing and analysis, editing GK data processing and analysis, editing

DS conceptualization, provided direction for the experimental approach, resources, supervision, wrote and critically revised the manuscript, obtained funding.

\section{Funding}

The project was supported by NIDA R0107418 and the JPB Foundation (DS). 


\section{References}

Albin, R. L., Young, A. B., \& Penney, J. B. (1989). The functional anatomy of basal ganglia disorders.

Bamford, N. S., Wightman, R. M., \& Sulzer, D. (2018, February 7). Dopamine's Effects on Corticostriatal Synapses during Reward-Based Behaviors. Neuron. Cell Press. https://doi.org/10.1016/j.neuron.2018.01.006

Cataldi, S., Stanley, A. T., Miniaci, M. C., \& Sulzer, D. (2021). Interpreting the role of the striatum during multiple phases of motor learning. The FEBS Journal, febs.15908. https://doi.org/10.1111/febs.15908

Coddington, L. T., \& Dudman, J. T. (2019). Learning from Action: Reconsidering Movement Signaling in Midbrain Dopamine Neuron Activity. Neuron, 104(1), 63-77. https://doi.org/10.1016/j.neuron.2019.08.036

DeLong, M. R. (1990). Primate models of movement disorders of basal ganglia origin. Trends in Neurosciences. Trends Neurosci. https://doi.org/10.1016/0166-2236(90)90110-V

Gangarossa, G., Espallergues, J., Mailly, P., De Bundel, D., de Kerchove d'Exaerde, A., Hervé, D., ... Krieger, P. (2013). Spatial distribution of D1R- and D2R-expressing medium-sized spiny neurons differs along the rostro-caudal axis of the mouse dorsal striatum. Frontiers in Neural Circuits, 7(JUL). https://doi.org/10.3389/fncir.2013.00124

Gerfen, C. R., Engber, T. M., Mahan, L. C., Susel, Z., Chase, T. N., Monsma, F. J., \& Sibley, D. R. (1990). D1 and D2 dopamine receptor-regulated gene expression of striatonigral and striatopallidal neurons. Science, 250(4986), 1429-1432. https://doi.org/10.1126/science. 2147780

Graybiel, A. M. (2008, June 17). Habits, rituals, and the evaluative brain. Annual Review of Neuroscience. Annual Reviews . https://doi.org/10.1146/annurev.neuro.29.051605.112851

Hunnicutt, B. J., Jongbloets, B. C., Birdsong, W. T., Gertz, K. J., Zhong, H., \& Mao, T. (2016). A comprehensive excitatory input map of the striatum reveals novel functional organization. ELife, 5(November2016). https://doi.org/10.7554/eLife.19103

Jin, X., Tecuapetla, F., \& Costa, R. M. (2014). Basal ganglia subcircuits distinctively encode the parsing and concatenation of action sequences. Nature Neuroscience, 17(3), 423-430. https://doi.org/10.1038/nn.3632

Kawaguchi, Y., Wilson, C. J., \& Emson, P. C. (1990). Projection subtypes of rat neostriatal 
matrix cells revealed by intracellular injection of biocytin. Journal of Neuroscience, 10(10), 3421-3438. https://doi.org/10.1523/jneurosci.10-10-03421.1990

Kravitz, A. V., Freeze, B. S., Parker, P. R. L., Kay, K., Thwin, M. T., Deisseroth, K., \& Kreitzer, A. C. (2010). Regulation of parkinsonian motor behaviours by optogenetic control of basal ganglia circuitry. Nature, 466(7306), 622-626. https://doi.org/10.1038/nature09159

Lingawi, N. W., \& Balleine, B. W. (2012). Amygdala central nucleus interacts with dorsolateral striatum to regulate the acquisition of habits. Journal of Neuroscience, 32(3), 1073-1081. https://doi.org/10.1523/JNEUROSCI.4806-11.2012

Mathis, A., Mamidanna, P., Cury, K. M., Abe, T., Murthy, V. N., Mathis, M. W., \& Bethge, M. (2018). DeepLabCut: markerless pose estimation of user-defined body parts with deep learning. Nature Neuroscience, 21(9), 1281-1289. https://doi.org/10.1038/s41593-0180209-y

Owesson-White, C., Belle, A. M., Herr, N. R., Peele, J. L., Gowrishankar, P., Carelli, R. M., \& Mark Wightman, R. (2016). Cue-evoked dopamine release rapidly modulates D2 neurons in the nucleus accumbens during motivated behavior. Journal of Neuroscience, 36(22), 60116021. https://doi.org/10.1523/JNEUROSCI.0393-16.2016

Silberberg, G., \& Bolam, J. P. (2015, August 1). Local and afferent synaptic pathways in the striatal microcircuitry. Current Opinion in Neurobiology. Elsevier Ltd. https://doi.org/10.1016/j.conb.2015.05.002

Vandaele, Y., Mahajan, N. R., Ottenheimer, D. J., Richard, J. M., Mysore, S. P., \& Janak, P. H. (2019). Distinct recruitment of dorsomedial and dorsolateral striatum erodes with extended training. ELife, 8. https://doi.org/10.7554/eLife.49536

Wall, N. R., DeLaParra, M., Callaway, E. M., \& Kreitzer, A. C. (2013). Differential innervation of direct- and indirect-pathway striatal projection neurons. Neuron, 79(2), 347-360. https://doi.org/10.1016/j.neuron.2013.05.014

Yin, H. H., Mulcare, S. P., Hilário, M. R. F., Clouse, E., Holloway, T., Davis, M. I., ... Costa, R. M. (2009). Dynamic reorganization of striatal circuits during the acquisition and consolidation of a skill. Nature Neuroscience, 12(3), 333-341. https://doi.org/10.1038/nn.2261 
bioRxiv preprint doi: https://doi.org/10.1101/2021.06.07.447452; this version posted June 8, 2021. The copyright holder for this preprint (which was not certified by peer review) is the author/funder, who has granted bioRxiv a license to display the preprint in perpetuity. It is made available under aCC-BY-NC-ND 4.0 International license. 


\section{Figures}

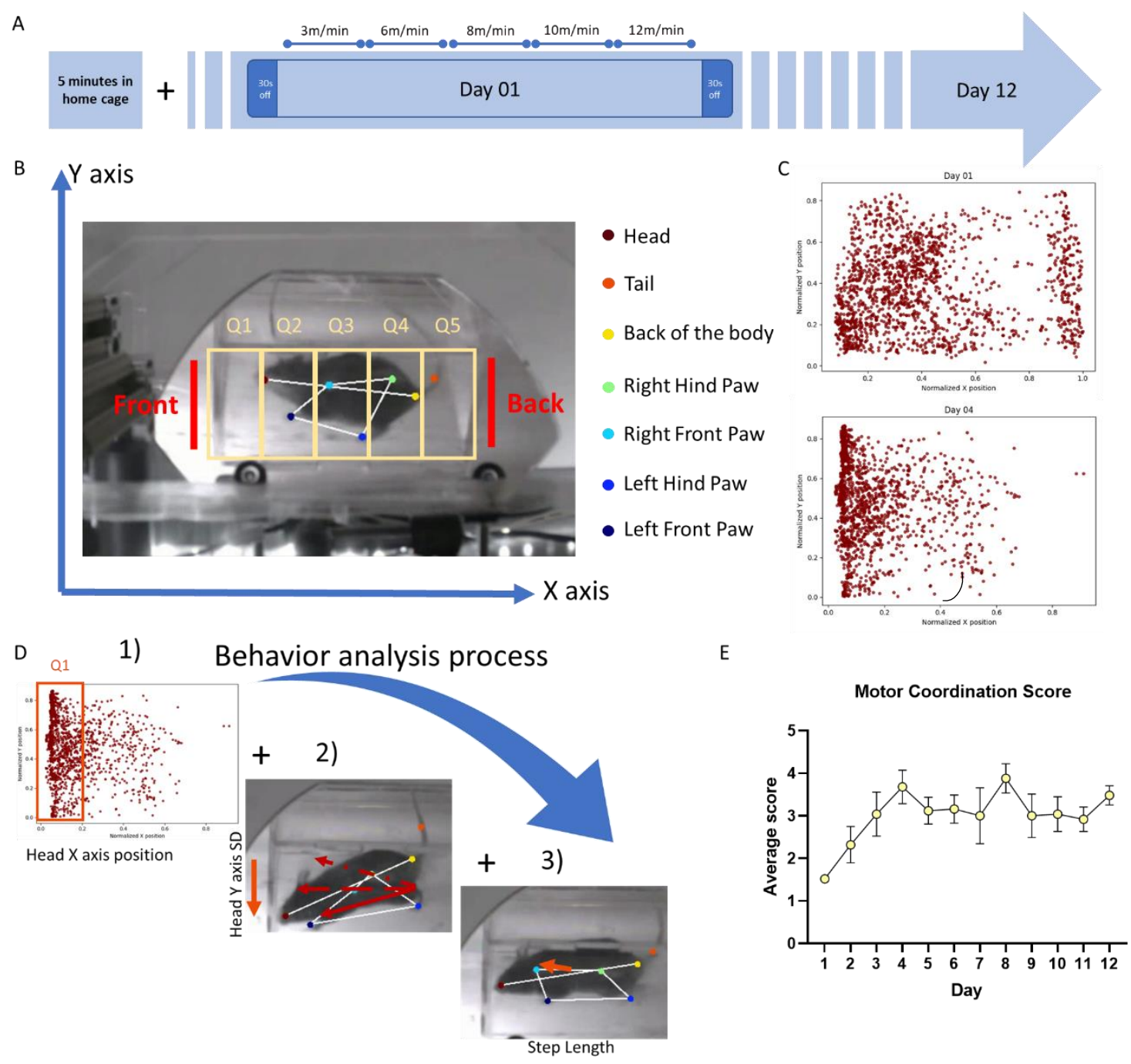

Figure 1. A) Timeline of behavioral protocol. On each experiment day mice are connected to the photometry patch cord in their home cage and a 5-minute calcium baseline signal is recorded. After this habituation period mice are placed on the treadmill and are left to explore the environment for 30 seconds. The treadmill motor is then started at a speed of $3 \mathrm{~m} / \mathrm{min}$, with increasing speed every 60 seconds, up to $12 \mathrm{~m} / \mathrm{min}$, after which the treadmill is turned off for another 30 seconds. This protocol is repeated for 12 consecutive days. B) Schematic of the behavioral analysis process. Position of mouse body parts is obtained through analysis with DeepLabCut. The 2D field of view is divided into five quadrants and the probability of the head of the mouse to be in each quadrant is calculated. Color code for the body part is indicated in the legend on the right. C) Samples of head positions in day 1 (above) and day 4 (below) for one control mouse (each dot represents the head position in one frame; all frames from one video are overlapped). On day 1 the head position if often towards the back of the field, indicating the mouse is falling behind the belt speed, and often hitting the back wall. By day 4 the animal is able to run and keep up with the treadmill, thus the position of the head is more consistently towards the front. D) Process for obtaining the mouse motor coordination score. Motor coordination score is calculated from head position (in the 5 quadrants), position along the $\mathrm{Y}$ axis (calculated from standard 
deviation of $Y$ axis value), and step length (distance between steps for each paw), as described in the Methods section. E) Motor coordination score for a cohort of control animals. Control mice show significant improvement in the coordination score over the 12 days of testing ( $n=5$; 1 -way ANOVA $* p<0.05)$.

A

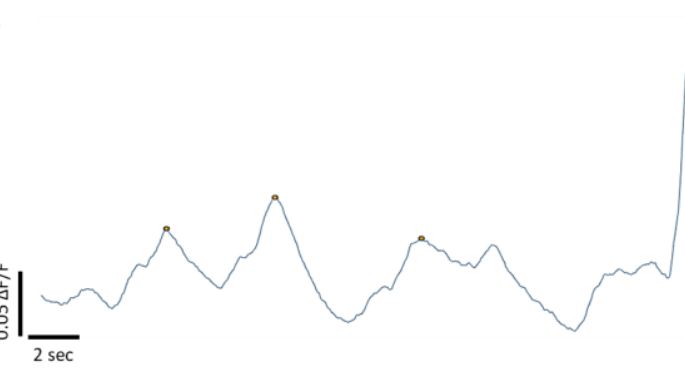

C.i. day 01

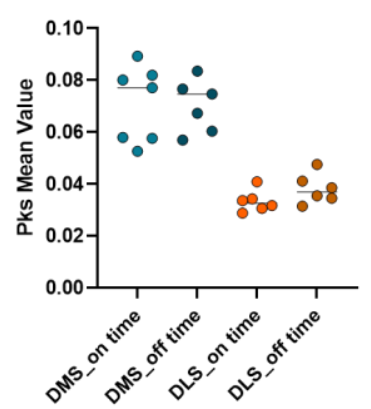

ii. day 03

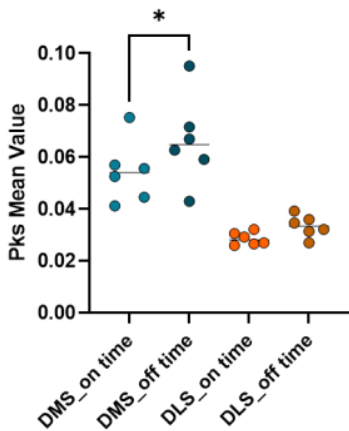

B

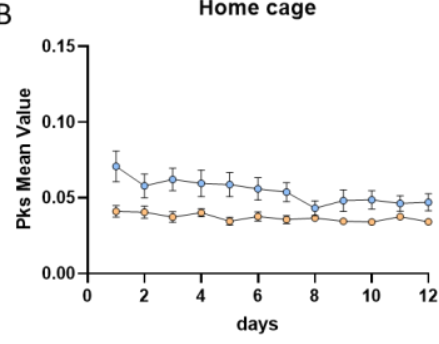

$\rightarrow$ DMS home cage

- DLS home cage

iii. day 07

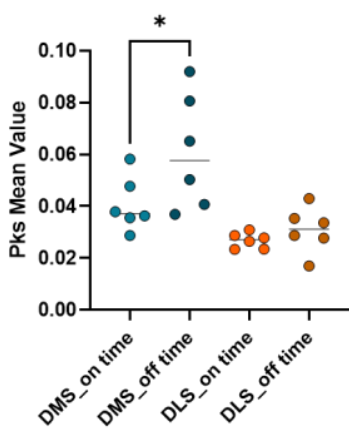

iv. day 12

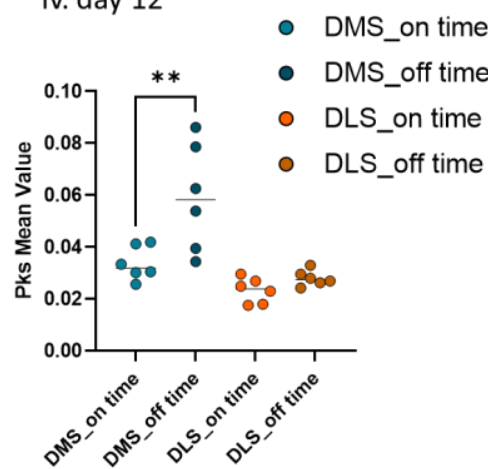

Figure 2. A) Sample trace of D1-SPNs calcium signaling recorded in the DMS of a mouse running on the treadmill. Calcium events are indicated by circles. B) Average event amplitude of D1-SPNs in the DMS (light blue) and DLS (light orange) from the daily 5 minutes home cage recording prior to treadmill testing. There is no significant reduction in event amplitude over the 12 days (1-way ANOVA $p=0.28$ for DMS and $p=0.52$ for DLS; $\mathrm{n}=6$ for each group). C) Average Peak Amplitude from treadmill recording. Running epochs at all treadmill speeds is averaged together and referred to as "on time". The 30 seconds prior to running and after the treadmill is turned off are averaged together and referred to as "off time". Day 1 (C.i.), day 3 (C.ii.), day 7 (C.iii.), and day 12 (C.iv.) are shown. There are no changes between on and off time for D1-SPNs recorded from the DLS ( $n=6$; day 01: 1-way ANOVA $p=0.43$, Bonferroni's multiple comparisons test $p=0.14$ DLS on-time vs DLS off-time; day 03: 1-way ANOVA $p<0.01$, Bonferroni's multiple comparisons test $p=0.06$ DLS on-time vs DLS off-time; day07: 1-way ANOVA $p<0.01$, Bonferroni's multiple comparisons test $p=0.63$ DLS on-time vs DLS off-time; day 12: 1-way ANOVA $p<0.0001$, Bonferroni's multiple comparisons test $p=0.95$ DLS on-time vs DLS off-time). DMS D1SPNs activity during on-time is comparable to off-time on day $01(n=6$; 1-way ANOVA $p=0.43$, Bonferroni's multiple comparisons test $p=0.79$ DMS on-time vs DMS off-time) and becomes significantly lower than off-time activity starting on day 3 (1-way ANOVA $p<0.01$, Bonferroni's multiple comparisons test $* p<0.05$ DMS on-time vs DMS off-time), and then on day 7 (1-way ANOVA $p<0.01$, Bonferroni's multiple comparisons test $* p<0.05$ DMS on-time vs DMS off-time) and day 12 (1-way ANOVA $p<0.0001$, Bonferroni's multiple comparisons test ** $p<0.01$ DMS on-time vs DMS off-time), suggesting that activity in this region is lower once the skill is acquired. 
A

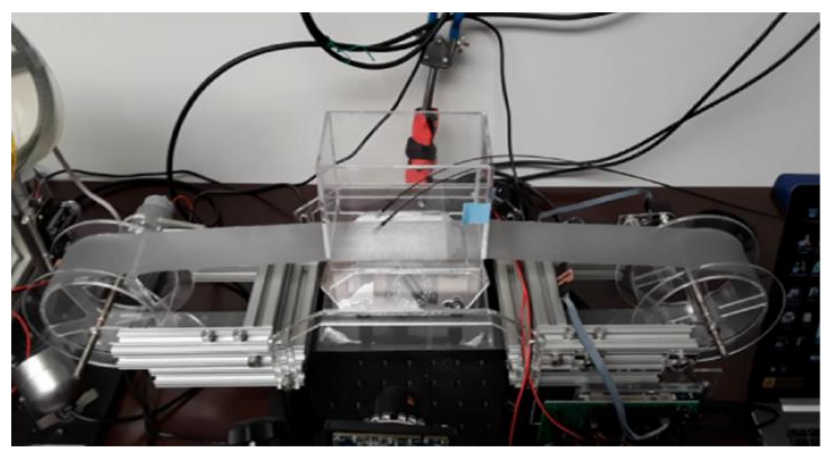

B

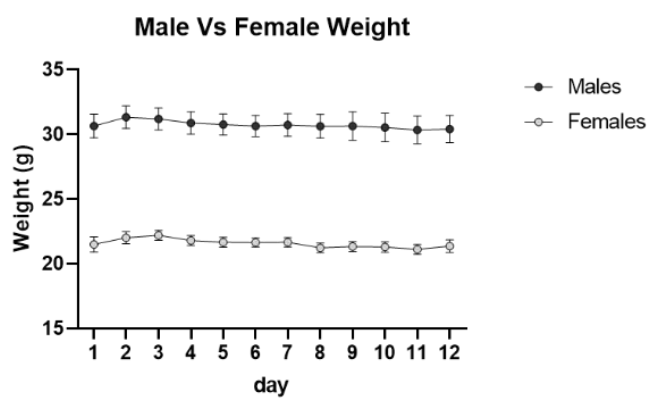

Supplementary Figure 1. A) Photo of the custom-built treadmill system. A tape is moved by a motor controlled via Arduino. A box is placed around the running area to prevent the mouse from jumping off the treadmill. A mirror is placed below the treadmill to visualize paw movement via a camera located on the side (not in picture). B) Mouse weight was recorded before each day of experiment. 12 consecutive days of training did not affect mouse weight (1-way ANOVA $p=0.90$ for time). Data showing differences between male and female weight at this age (3-4 months of age; $n=10$ males; $n=7$ females; 2 -way ANOVA $p<0.0001)$.

A

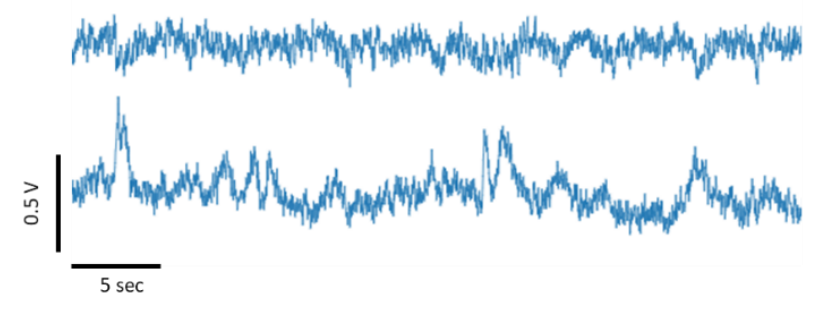

C

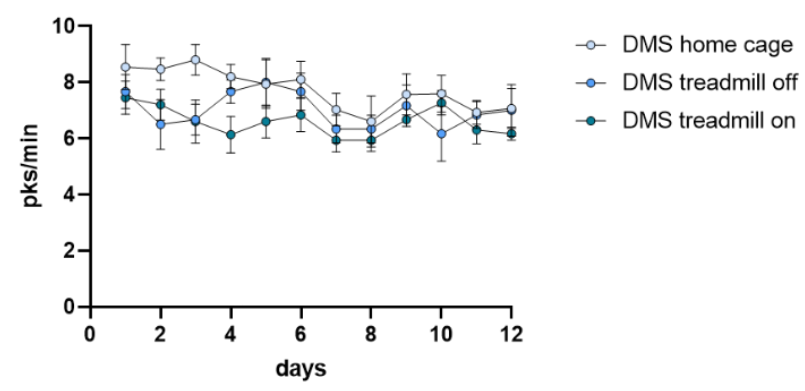

B

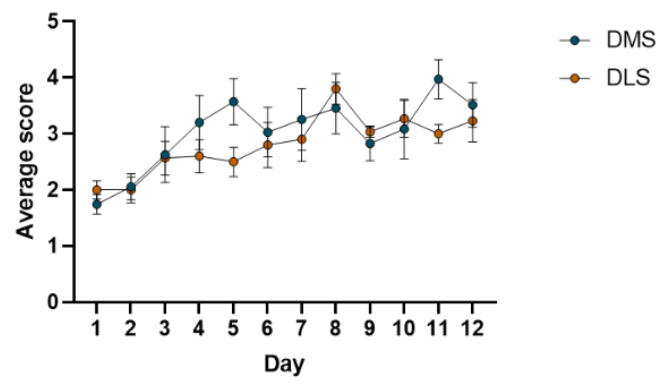

D

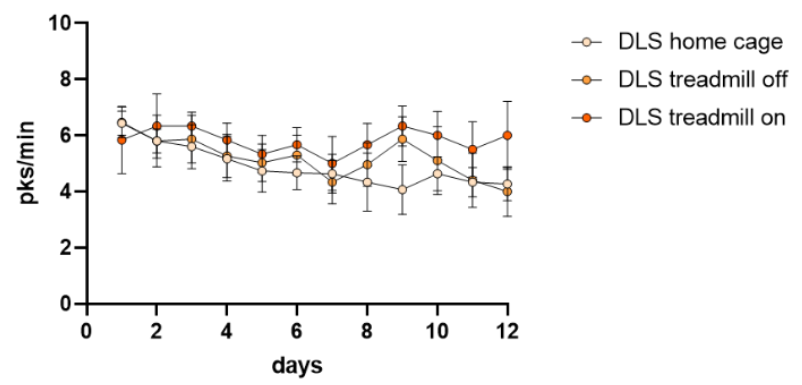

Supplementary Figure 2. A) Raw sample trace showing no movement artifact as recorded by $405 \mathrm{~nm}$, excitation wavelength for GCaMP6f isosbestic point (top trace), in comparison to calcium signal (bottom trace). B) Coordination score from treadmill running for DMS and DLS injected animals. Mice perform similarly to control animals, indicating that surgery, viral expression, and optic fiber implant do not affect running abilities and motor learning (1-way ANOVA * $p<0.05$ for DMS mice and $* * p<0.01$ for DLS mice). C,D) Average number of calcium events for D1-SPNs in the DMS (C) and DLS (D). Measurements 
for epochs of running time (treadmill on), time in which the treadmill is off, and peak counts in home cage baseline recording are overlapped. There are no significant changes in the average number of peaks over the 12 days of training for DMS (2-way ANOVA Time $x$ Column Factor $p=0.82$ ) or DLS recording (2-way ANOVA Time $x$ Column Factor $p=0.77$ for DLS).
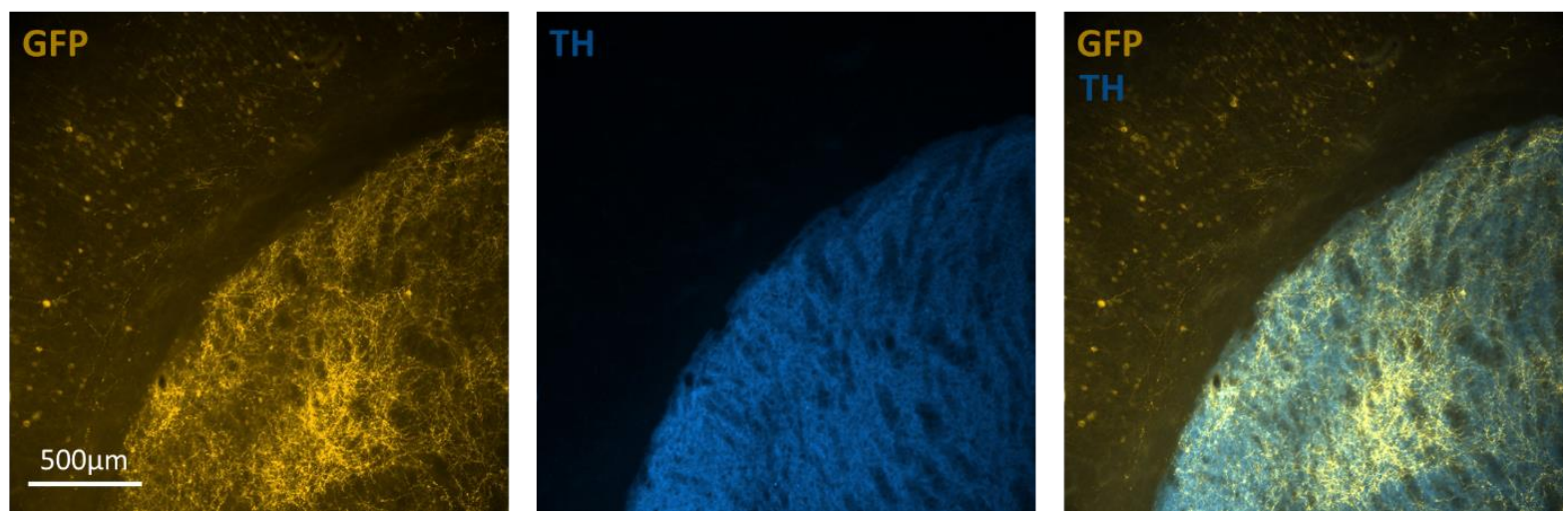

Supplementary Figure 3. Sample pictures of coronal sections of the dorsal striatum of D1-cre animals injected with AAV vectors containing Flex-GCaMP6f. GCaMP6f expression is confirmed by GFP immunolabeling (yellow) co-stained with TH antibody (blue) to visualize the striatum. 